\title{
Murine Depression Model and its Potential Applications for Discovering Foods and Farm Products with Antidepressant-Like Effects
}

\author{
Tatsuhiko Goto ${ }^{1,2}$, Shozo Tomonaga ${ }^{3}$, Tsuyoshi Okayama ${ }^{1,2,4}$ and Atsushi Toyoda 1,2,4* \\ ${ }^{1}$ Department of Biological Production Science, College of Agriculture, Ibaraki University, Ami, Ibaraki, Japan, ${ }^{2}$ Department of \\ Biological Production Science, Ibaraki University Cooperation between Agriculture and Medical Science, Ami, Ibaraki, Japan, \\ ${ }^{3}$ Graduate School of Agriculture, Kyoto University, Kyoto, Japan, ${ }^{4}$ Department of Biological Production Science, United \\ Graduate School of Agricultural Science, Tokyo University of Agriculture and Technology, Fuchu, Japan
}

\section{OPEN ACCESS}

Edited by:

Fumihiko Maekawa,

National Institute for Environmental

Studies, Japan

Reviewed by:

Bruno Bonaz,

Grenoble Faculty of Medicine and

Hospital, France

Keizo Takao,

University of Toyama, Japan

Adriana Ximenes-da-Silva,

Universidade Federal de Alagoas,

Brazil

*Correspondence: Atsushi Toyoda atsushi.toyoda.0516@vc.ibaraki.ac.jp

Specialty section:

This article was submitted to Neuroendocrine Science,

a section of the journal

Frontiers in Neuroscience

Received: 07 December 2015

Accepted: 16 February 2016

Published: 01 March 2016

Citation:

Goto T, Tomonaga S, Okayama T and Toyoda A (2016) Murine Depression Model and its Potential Applications for Discovering Foods and Farm

Products with Antidepressant-Like Effects. Front. Neurosci. 10:72. doi: 10.3389/fnins.2016.00072
Advanced societies face increased health problems related to various stresses. Chronic psychological stress is a major risk factor for psychiatric disorders such as depression. Although therapeutic agents reduce several symptoms of depression, most have side effects in a broad range of the population. Furthermore, some victims of depression do not show significant improvement with any drugs, so alternative approaches are needed. Good dietary habits may potentially reduce depressive symptoms, but there is little scientific evidence thus far. Murine depression models are useful to test nutritional approaches in vivo. Our model mice subjected to a subchronic mild social defeat stress (SCSDS) paradigm show several alterations in physiological parameters and social behavior. These stress-induced symptoms in SCSDS mice can be used as cues to identify antidepressant-like natural resources including foods and farm products. We previously discovered that SCSDS mice show more vulnerability to social stress by changing dietary condition. In addition, we developed a more objective system for analyzing mouse behavior using a 3D depth-sensing camera to understand relationships between diet and behavior. The combination of SCSDS mice with 3D behavioral analysis is a powerful method for screening ingredients in foods and farm products for antidepressant-like effects.

Keywords: depression, food, behavior, metabolomics, social defeat stress, depth camera

\section{INTRODUCTION}

Advanced societies face increased health problems related to various stresses; chronic psychological stress in particular is a major risk factor for precipitating psychiatric disorders such as depression. A Global Burden of Disease study showed depression is the most disabling disorder worldwide (Whiteford et al., 2013). Since this issue will face the next generation of developing countries, it is imperative to find some solution. Therapeutic agents reduce several symptoms of depression, but most have several side effects in a broad population (Stevens et al., 2014; Galling et al., 2015). Moreover, some victims of depression do not show significant improvement with any 
drugs (treatment-resistant depression), so alternative approaches are needed (El-Hage et al., 2013). For prevention, it is more important to change dietary habits rather than resort to conventional treatments. Recently, interest has increased in Kampo (Watanabe et al., 2011) and functional foods (Arai, 1996). In fact, some reports indicate that Kampo (Ito et al., 2012; Hori et al., 2015) and functional ingredients (Tomonaga et al., 2008; Iio et al., 2012a) have antidepressant effects in animal models. This study further explores functional ingredients from natural sources for prevention and attenuation of symptoms of depression.

Chronic social defeat stress (CSDS) models of rats and mice are recognized as good animal models of depression. CSDS models establish social stress using male territorial aggression (Kudryavtseva et al., 1991; Miczek et al., 2008; Hammels et al., 2015). We previously studied CSDS rats and found that social stress induces alterations in the MAP kinase cascade, hypothalamic malonyl-CoA, peripheral leptin, digestive system, and behaviors (Iio et al., 2011, 2012b, 2014; Toyoda et al., 2015). Others have analyzed both central nervous system and peripheral tissues in relation to stress resilience using CSDS mice (Russo and Nestler, 2013; Hodes et al., 2014; Pfau and Russo, 2015). In addition, we have established a milder model of depression, subchronic mild social defeat stress (sCSDS; Goto et al., 2014, Goto and Toyoda, 2015) than CSDS (Krishnan et al., 2007). Since several models are required (Bartolomucci and Leopardi, 2009), sCSDS mice may provide insights about pathogenic mechanisms and preventive measures for depression.

The 3D behavioral analysis allows evaluation of more natural, realistic animal behavior. Although animals behave in $3 \mathrm{D}$ space and show several postures sterically, conventional animal monitoring systems have been primarily $2 \mathrm{D}$ video cameras. Recently, commercially available 3D depth-sensing cameras have been developed in the computer game industry, and can thus be affordably obtained ( $\sim 200 /$ camera). Using 3D cameras, rats and mice have been monitored (Ou-Yang et al., 2011). Since behavioral scientists can derive depth information from animal behavior, the 3D camera enables discrimination of slight differences in $3 \mathrm{D}$ behavioral characteristics that have not been previously well detected.

In this article, we introduce features of our murine stress model and novel 3D monitoring system. We discuss the use of both for discovering foods and farm products providing antidepressant-like effects.

\section{SUBCHRONIC MILD SOCIAL DEFEAT STRESS (SCSDS) MODEL MOUSE \\ Phenotypes of sCSDS (Figure 1A)}

sCSDS mice are established by the method (Goto and Toyoda, 2015). Subject C57BL/6J mice are exposed to psychosocial stress from aggressive ICR mice for 10 consecutive days. During establishment, body weight gain, food intake, and water intake in sCSDS mice are significantly higher than those of nonstressed control mice (Goto et al., 2014). The sCSDS mice show increased body water content and social avoidance behavior after the stress. Moreover, nest-building behavior in sCSDS mice is significantly delayed compared to control mice (Otabi et al., 2016). In CSDS mice, social avoidance behavior has been widely reported (Tanaka et al., 2012; Russo and Nestler, 2013). Increased body weight gain has been reported in defeated mice (Goto et al., 2014) and lower ranking mice (Kim et al., 2015). Stress-induced polydipsia has been reported in both CSDS (Krishnan et al., 2007) and chronic mild stress (CMS) conditions (Gross and Pinhasov, 2016). Although, these features are supported by several studies, social stress-induced increases in body water content (Goto et al., 2014) and delays in nestbuilding behavior (Otabi et al., 2016) are, to our knowledge, unique findings.

\section{Metabolomic Analyses of sCSDS (Figure 1A)}

To find key metabolites altered by social defeat stress, we tested the blood plasma/serum, urine, and liver. Biochemical assays for blood serum components revealed that sCSDS mice showed low levels of albumin (ALB) and blood urea nitrogen (BUN) just after stress (Goto et al., 2014). Low levels of sodium were found in the urine of sCSDS mice. Metabolomics revealed that four liver metabolites, taurocyamine (GES), phosphorylcholine, D-alanylD-alanine (D-ala-D-ala), and 1-methylnicotinamide (MNA), were significantly upregulated in sCSDS mice vs. control mice (Goto et al., 2015a).

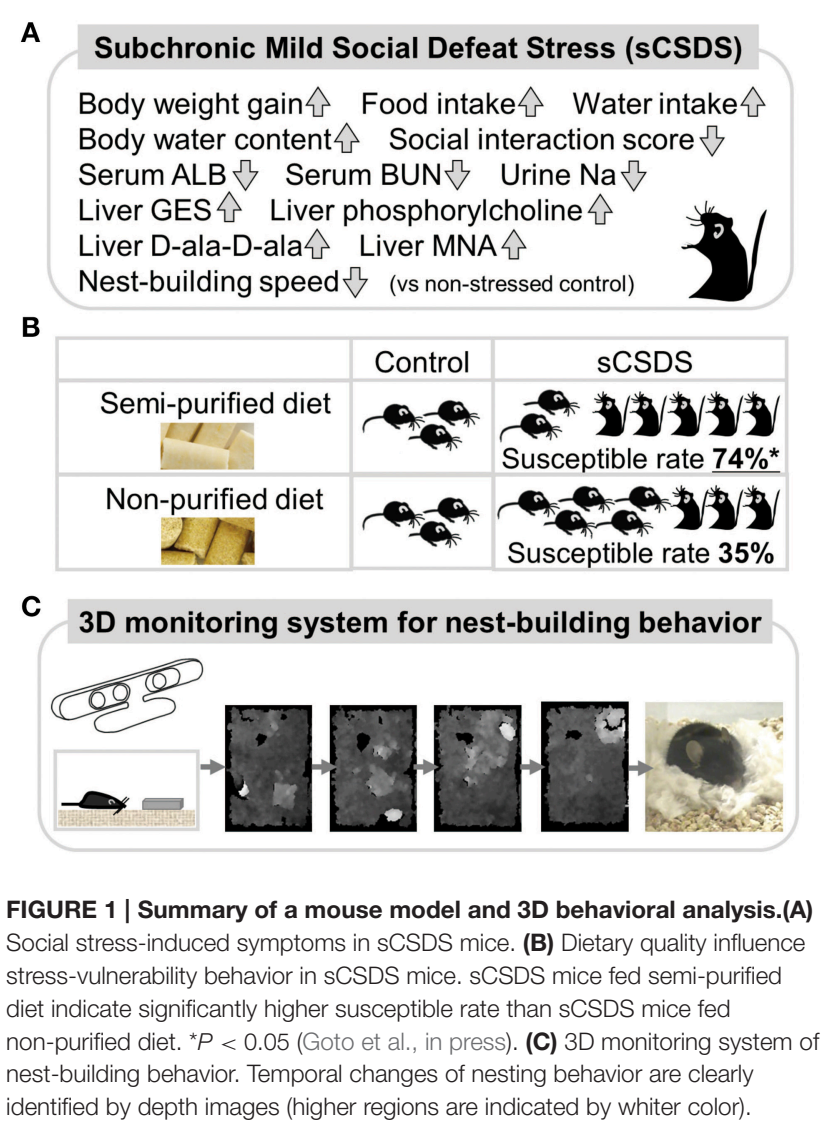




\section{Food Habits Attenuate Stress-Induced Symptoms in sCSDS (Figure 1B)}

We established sCSDS mice under feeding conditions with two kinds of pellet food, a semi-purified and a non-purified diet. We confirmed that the increased body weight gain, food intake, and water intake of sCSDS mice during stress were common to both diets (Goto et al., in press). Interestingly, we found that the vulnerability of mice to social defeat stress was affected by diet quality. sCSDS mice fed a semi-purified diet were more susceptible than sCSDS mice fed a non-purified diet (Goto et al., in press). This may be due to changing gut environments, as gut microbiota and their metabolic products can affect animal brain function and behavior (Cryan and Dinan, 2012). Especially, commensal microbiota can influence the hypothalamus-pituitary-adrenal reaction to stress in mice (Sudo et al., 2004). And, gut microbiota can modulate brain development and modulation of the serotonergic system, which is directly related to mood, in the limbic system (Heijtz et al., 2011). Although the microflora of sCSDS mice have not been fully studied, intestinal flora will be influenced by both stress and food. Metabolomics with the sCSDS mice fed both diets hope to find some solutions to attenuate stress-induced behavior by changing the metabolic environments of peripheral tissues.

\section{A NOVEL 3D BEHAVIORAL TESTING METHOD FOR INNATE BEHAVIOR (FIGURE 1C)}

Infrared 3D depth-sensing cameras have been developed intensely in the computer game industry. Both Microsoft Kinect (Microsoft Corp., USA) and Xtion PRO LIVE (ASUSTek Computer Inc., Taiwan) have become commercially available and affordable. The 3D depth-sensing camera has thus recently become available for use in rodent behavioral tests. The first reported behavioral analyses using a $3 \mathrm{D}$ camera focused on locomotion and pose in rats and mice (Ou-Yang et al., 2011). Matsumoto et al. constructed skeleton models of rats using four 3D cameras simultaneously and analyzed social and sexual interactions and novel object recognition behavior (Matsumoto et al., 2013, 2014). We monitored nest-building behavior in mice using a 3D camera (Okayama et al., 2015), and confirmed its effective utilization in a genetic study (Goto et al., 2015b). Nakamura et al. have developed a gait analysis system for mice using a 3D camera (Nakamura et al., 2015). Hong et al. succeeded in automating measurement of mouse social behaviors with machine learning algorithms from images captured simultaneously by a $3 \mathrm{D}$ camera and two $2 \mathrm{D}$ video cameras (Hong et al., 2015). Although conventional testing has been performed with $2 \mathrm{D}$ video cameras, future animal behavioral testing will evolve dramatically through use of $3 \mathrm{D}$ cameras.

Our 3D monitoring system focused on nesting behavior in mice, because the steric nest can be evaluated as a visible behavior. Deacon codified a standard method of nest-building behavior in mice using pressed cotton and rated nest quality on a scale of 1-5 (Deacon, 2006). By utilizing 3D depth-sensing cameras, we are able to conduct objective $3 \mathrm{D}$ evaluation of the final nest in one point evaluation (Okayama et al., 2015). In addition, we have analyzed an untapped behavioral characteristic, the construction process of the nest. Our 3D monitoring system could discriminate slight differences in temporal nesting behavior using 3D depth images and Deacon score 1-5 (Goto et al., 2015b). Since nest-building behavior is altered by social stress (Otabi et al., 2016), several stress models such as CSDS, CMS, and restraint stress should also be analyzed while nest-building in future.

\section{STRATEGY FOR FINDING ANTIDEPRESSANTS FROM NATURAL INGREDIENTS (FIGURE 2)}

Using sCSDS mice, we will investigate functional ingredients from natural sources that should attenuate stress-induced symptoms. Since sCSDS mice fed a semi-purified diet show more vulnerable behavior than mice fed a non-purified diet (Goto et al., in press), a change to a semi-purified diet as a base diet would be a good approach to identify functional ingredients enhancing stress resilience behavior. A semi-purified diet is preferred for nutritional research because the non-purified diet contains unknown raw materials and ingredients. Since the non-purified diet potentially improves stress vulnerability, it would be worthwhile to note differences between semi-purified and non-purified diets. Components including dietary fiber, resistant starch, and unavailable carbohydrates will be targets in future studies. These components are available to gut microbiota, resulting in a variety of species living in the gut. The braingut axis (Kelly et al., 2015) should be a main target for foodmediated approaches to finding antidepressants and preventing stress-related diseases by shifting food habits. In addition, sCSDS mice show stress-induced symptoms described above. By checking these indicators, the depression model can be useful for screening antidepressant effects of functional food and farm products.

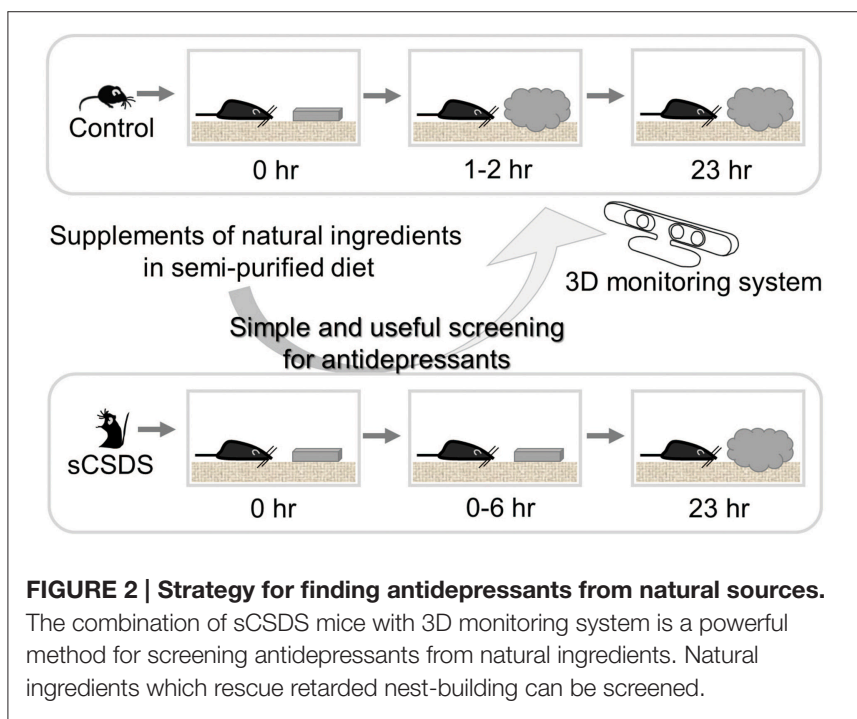


Our novel 3D monitoring system for nest-building enabled us to detect small differences among groups in different treatments and strains (Okayama et al., 2015; Goto et al., 2015b). Furthermore, we found simple and useful nesting behavioral test (Otabi et al., 2016). By using this combined method with sCSDS mice and 3D monitoring systems, we can discern slight differences in innate behavior and screen candidate supplements quickly. In future, automated behavioral analysis for nesting behavior will make this method easy for many researchers.

Toyoda and colleagues conducted research in the interdisciplinary field of agricultural-medical science (http:// iucam-ibaraki.wix.com/iucam), exploring functional food and farm products for prevention and attenuation of psychiatric symptoms. Establishing animal models of depression, searching and screening supplemental resources, and applying engineering techniques will be essential in the big data era. Now that the fundamental platform has been established, we can screen ingredients from natural sources such as dairy products, fruits, and vegetables.

\section{CONCLUSIONS}

In this article, we introduced our mouse model of depression and novel 3D evaluation system for nesting behavior. Good dietary habits should provide potential effects for

\section{REFERENCES}

Arai, S. (1996). Studies on functional foods in Japan-state of the art. Biosci. Biotechnol. Biochem. 60, 9-15. doi: 10.1271/bbb.60.9

Bartolomucci, A., and Leopardi, R. (2009). Stress and depression: preclinical research and clinical implications. PLOS ONE 4:e4265. doi: 10.1371/journal.pone.0004265

Cryan, J. F., and Dinan, T. G. (2012). Mind-altering microorganisms: the impact of the gut microbiota on brain and behaviour. Nat. Rev. Neurosci. 13, 701-712. doi: $10.1038 / \mathrm{nrn} 3346$

Deacon, R. M. J. (2006). Assessing nest building in mice. Nat. Protoc. 1, 1117-1119. doi: 10.1038/nprot.2006.170

El-Hage, W., Leman, S., Camus, V., and Belzung, C. (2013). Mechanisms of antidepressant resistance. Front. Pharmacol. 4:146. doi: 10.3389/fphar.2013.00146

Galling, B., Calsina Ferrer, A., Abi Zeid Daou, M., Sangroula, D., Hagi, K., and Correll, C. U. (2015). Safety and tolerability of antidepressant cotreatment in acute major depressive disorder: results from a systematic review and exploratory meta-analysis. Expert Opin. Drug Saf. 14, 1587-1608. doi: 10.1517/14740338.2015.1085970

Goto, T., Kubota, Y., Tanaka, Y., Iio, W., Moriya, N., and Toyoda, A. (2014). Subchronic and mild social defeat stress accelerates food intake and body weight gain with polydipsia-like features in mice. Behav. Brain Res. 270, 339-348. doi: 10.1016/j.bbr.2014.05.040

Goto, T., Kubota, Y., and Toyoda, A. (2015a). Plasma and liver metabolic profiles in mice subjected to subchronic and mild social defeat stress. J. Proteome Res. 14, 1025-1032. doi: 10.1021/pr501044k

Goto, T., Kubota, Y., and Toyoda, A. (in press). Effects of diet quality on vulnerability to mild subchronic social defeat stress in mice. Nutr. Neurosci. doi: 10.1179/1476830515y.0000000017

Goto, T., Okayama, T., and Toyoda, A. (2015b). Strain differences in temporal changes of nesting behaviors in $\mathrm{C} 57 \mathrm{BL} / 6 \mathrm{~N}, \mathrm{DBA} / 2 \mathrm{~N}$, and their $\mathrm{F} 1$ hybrid mice assessed by a three-dimensional monitoring system. Behav. Processes 119, 86-92. doi: 10.1016/j.beproc.2015.07.007 reducing depressive symptoms and extending healthy life. The importance of food habits in overcoming stress can be shown by identifying antidepressant-like natural resources using sCSDS mice. The combination of sCSDS mice with $3 \mathrm{D}$ behavioral assays is a powerful method for screening the ingredients showing antidepressant-like effects in foods and farm products.

\section{AUTHOR CONTRIBUTIONS}

TG and AT wrote overall manuscript. ST and TO wrote a part of the manuscript about metabolome and $3 \mathrm{D}$ sensor, respectively. TG, ST, TO, and AT have checked the manuscript entirely and agree with submission.

\section{FUNDING}

This research was supported in part by an Ibaraki University Cooperation between Agriculture and Medical Science (IUCAM) (The MEXT, Japan) and the Council for Science, Technology and Innovation (CSTI) under the Cross-ministerial Strategic Innovation Promotion Program (SIP) “Technologies for creating next-generation agriculture, forestry, and fisheries" (Bio-oriented Technology Research Advancement Institution, NARO) (The Cabinet Office, Japan).

Goto, T., and Toyoda, A. (2015). A mouse model of subchronic and mild social defeat stress for understanding stress-induced behavioral and physiological deficits. J. Vis. Exp. 105:e52973. doi: 10.3791/52973

Gross, M., and Pinhasov, A. (2016). Chronic mild stress in submissive mice: marked polydipsia and social avoidance without hedonic deficit in the sucrose preference test. Behav. Brain Res. 298, 25-34. doi: 10.1016/j.bbr.2015. 10.049

Hammels, C., Pishva, E., De Vry, J., van den Hove, D. L. A., Prickaerts, J., van Winkel, R., et al. (2015). Defeat stress in rodents: From behavior to molecules. Neurosci. Biobehav. Rev. 59, 111-140. doi: 10.1016/j.neubiorev.2015.10.006

Heijtz, R. D., Wang, S., Anuar, F., Qian, Y., Bjorkholm, B., Samuelsson, A., et al. (2011). Normal gut microbiota modulates brain development and behavior. Proc. Natl. Acad. Sci. U.S.A. 108, 3047-3052. doi: 10.1073/pnas.1010 529108

Hodes, G. E., Pfau, M. L., Leboeuf, M., Golden, S. A., Christoffel, D. J., Bregman, D., et al. (2014). Individual differences in the peripheral immune system promote resilience versus susceptibility to social stress. Proc. Natl. Acad. Sci. U.S.A. 111, 16136-16141. doi: 10.1073/pnas.1415191111

Hong, W., Kennedy, A., Burgos-Artizzu, X. P., Zelikowsky, M., Navonne, S. G., Perona, P., et al. (2015). Automated measurement of mouse social behaviors using depth sensing, video tracking, and machine learning. Proc. Natl. Acad. Sci. U.S.A. 112, E5351-E5360. doi: 10.1073/pnas.1515982112

Hori, A., Ito, N., Oikawa, T., and Hanawa, T. (2015). Kososan, but not milnacipran, elicits antidepressant-like effects in a novel psychological stress-induced mouse model of depression. Tradit. Kampo Med. 2, 1-7. doi: 10.1002/tkm2.1013

Iio, W., Matsukawa, N., Tsukahara, T., Kohari, D., and Toyoda, A. (2011). Effects of chronic social defeat stress on MAP kinase cascade. Neurosci. Lett. 504, 281-284. doi: 10.1016/j.neulet.2011.09.047

Iio, W., Matsukawa, N., Tsukahara, T., and Toyoda, A. (2012a). The effects of oral taurine administration on behavior and hippocampal signal transduction in rats. Amino Acids 43, 2037-2046. doi: 10.1007/s00726-012-1282-2

Iio, W., Takagi, H., Ogawa, Y., Tsukahara, T., Chohnan, S., and Toyoda, A. (2014). Effects of chronic social defeat stress on peripheral leptin and its hypothalamic actions. BMC Neurosci. 15:72. doi: 10.1186/1471-2202-15-72 
Iio, W., Tokutake, Y., Matsukawa, N., Tsukahara, T., Chohnan, S., and Toyoda, A. (2012b). Anorexic behavior and elevation of hypothalamic malonyl-CoA in socially defeated rats. Biochem. Biophys. Res. Commun. 421, 301-304. doi: 10.1016/j.bbrc.2012.04.004

Ito, N., Hori, A., Yabe, T., Nagai, T., Oikawa, T., Yamada, H., et al. (2012). Involvement of neuropeptide $\mathrm{Y}$ signaling in the antidepressant-like effect and hippocampal cell proliferation induced by kososan, a Kampo medicine, in the stress-induced depression-like model mice. Biol. Pharm. Bull. 35, 1775-1783. doi: 10.1248/bpb.b12-00466

Kelly, J. R., Kennedy, P. J., Cryan, J. F., Dinan, T. G., Clarke, G., and Hyland, N. P. (2015). Breaking down the barriers: the gut microbiome, intestinal permeability and stress-related psychiatric disorders. Front. Cell. Neurosci. 9:392. doi: 10.3389/fncel.2015.00392

Kim, B., Colon, E., Chawla, S., Vandenberg, L. N., and Suvorov, A. (2015). Endocrine disruptors alter social behaviors and indirectly influence social hierarchies via changes in body weight. Environ. Health 14:64. doi: 10.1186/s12940-015-0051-6

Krishnan, V., Han, M.-H., Graham, D. L., Berton, O., Renthal, W., Russo, S. J., et al. (2007). Molecular adaptations underlying susceptibility and resistance to social defeat in brain reward regions. Cell 131, 391-404. doi: 10.1016/j.cell.2007.09.018

Kudryavtseva, N. N., Bakshtanovskaya, I. V., and Koryakina, L. A. (1991). Social model of depression in mice of C57BL/6J strain. Pharmacol. Biochem. Behav. 38, 315-320. doi: 10.1016/0091-3057(91)90284-9

Matsumoto, J., Uehara, T., Urakawa, S., Takamura, Y., Sumiyoshi, T., Suzuki, M., et al. (2014). 3D video analysis of the novel object recognition test in rats. Behav. Brain Res. 272, 16-24. doi: 10.1016/j.bbr.2014.06.047

Matsumoto, J., Urakawa, S., Takamura, Y., Malcher-Lopes, R., Hori, E., Tomaz, C., et al. (2013). A 3D-video-based computerized analysis of social and sexual interactions in rats. PLOS ONE 8:e78460. doi: 10.1371/journal.pone.00 78460

Miczek, K. A., Yap, J. J., and Covington, H. E. (2008). Social stress, therapeutics and drug abuse: preclinical models of escalated and depressed intake. Pharmacol. Ther. 120, 102-128. doi: 10.1016/j.pharmthera.2008. 07.006

Nakamura, A., Funaya, H., Uezono, N., Nakashima, K., Ishida, Y., Suzuki, T., et al. (2015). Low-cost three-dimensional gait analysis system for mice with an infrared depth sensor. Neurosci. Res. 100, 55-62. doi: 10.1016/j.neures.2015.06.006

Okayama, T., Goto, T., and Toyoda, A. (2015). Assessing nest-building behavior of mice using a 3D depth camera. J. Neurosci. Methods 251, 151-157. doi: 10.1016/j.jneumeth.2015.05.019

Otabi, H., Goto, T., Okayama, T., Kohari, D., and Toyoda, A. (2016). Subchronic and mild social defeat stress alter mouse nest building behavior. Behav. Processes 122, 21-25. doi: 10.1016/j.beproc.2015.10.018
Ou-Yang, T.-H., Tsai, M.-L., Yen, C.-T., and Lin, T.-T. (2011). An infrared range camera-based approach for three-dimensional locomotion tracking and pose reconstruction in a rodent. J. Neurosci. Methods 201, 116-123. doi: 10.1016/j.jneumeth.2011.07.019

Pfau, M. L., and Russo, S. J. (2015). Peripheral and central mechanisms of stress resilience. Neurobiol. Stress 1, 66-79. doi: 10.1016/j.ynstr.2014.09.004

Russo, S. J., and Nestler, E. J. (2013). The brain reward circuitry in mood disorders. Nat. Rev. Neurosci. 14, 609-625. doi: 10.1038/nrn3381

Stevens, J. R., Jarrahzadeh, T., Brendel, R. W., and Stern, T. A. (2014). Strategies for the prescription of psychotropic drugs with black box warnings. Psychosomatics 55, 123-133. doi: 10.1016/j.psym.2013.08.009

Sudo, N., Chida, Y., Aiba, Y., Sonoda, J., Oyama, N., Yu, X.-N., et al. (2004). Postnatal microbial colonization programs the hpothalamic-pituitaryadrenal system for stress response in mice. J. Physiol. 558, 263-275. doi: 10.1113/jphysiol.2004.063388

Tanaka, K., Furuyashiki, T., Kitaoka, S., Senzai, Y., Imoto, Y., Segi-Nishida, E., et al. (2012). Prostaglandin E2-mediated attenuation of mesocortical dopaminergic pathway is critical for susceptibility to repeated social defeat stress in mice. J. Neurosci. 32, 4319-4329. doi: 10.1523/JNEUROSCI.5952-11.2012

Tomonaga, S., Yamane, H., Onitsuka, E., Yamada, S., Sato, M., Takahata, Y., et al. (2008). Carnosine-induced antidepressant-like activity in rats. Pharmacol. Biochem. Behav. 89, 627-632. doi: 10.1016/j.pbb.2008.02.021

Toyoda, A., Iio, W., Matsukawa, N., and Tsukahara, T. (2015). Influence of chronic social defeat stress on digestive system functioning in rats. J. Nutr. Sci. Vitaminol. 61, 280-284. doi: 10.3177/jnsv.61.280

Watanabe, K., Matsuura, K., Gao, P., Hottenbacher, L., Tokunaga, H., Nishimura, K., et al. (2011). Traditional Japanese Kampo Medicine: clinical research between modernity and traditional medicine-the state of research and methodological suggestions for the future. Evid. Based Complement. Alternat. Med. 2011:513842. doi: 10.1093/ecam/neq067

Whiteford, H. A., Degenhardt, L., Rehm, J., Baxter, A. J., Ferrari, A. J., Erskine, H. E., et al. (2013). Global burden of disease attributable to mental and substance use disorders: findings from the Global Burden of Disease Study 2010. Lancet 382, 1575-1586. doi: 10.1016/S0140-6736(13)61611-6

Conflict of Interest Statement: The authors declare that the research was conducted in the absence of any commercial or financial relationships that could be construed as a potential conflict of interest.

Copyright (c) 2016 Goto, Tomonaga, Okayama and Toyoda. This is an open-access article distributed under the terms of the Creative Commons Attribution License (CC $B Y)$. The use, distribution or reproduction in other forums is permitted, provided the original author(s) or licensor are credited and that the original publication in this journal is cited, in accordance with accepted academic practice. No use, distribution or reproduction is permitted which does not comply with these terms. 